\title{
Clinical Impact of two Different Diagnostic Strategies in the First- and Second-Line Treatment of Locally Advanced or Metastatic EGFR-Mutated Non-Small Cell Lung Cancer
}

\author{
Giovanni Gancitano 1, Roberto Ravasio 2, Lorenzo Cattelino 1, Paolo Di Procolo ${ }^{1}$, \\ Diego Cortinovis ${ }^{3}$ \\ Medical \& Market Access Department, Roche Diagnostics SpA, Monza, Italy \\ Health Publishing \& Services Srl, Milano, Italy \\ SC Oncologia Medica, Ospedale San Gerardo, Monza, Italy
}

\begin{abstract}
BACKGROUND: A histopathological and mutational diagnosis has become a priority in the correct choice of the most appropriate cancer therapy for NSCLC. In the absence of a molecular analysis, the therapeutic choice will be directed towards platinum-based chemotherapy, thus preventing, in the presence of a specific mutation, the benefits deriving from the administration of a target therapies (TT).

AIM: the present analysis was carried out with the aim of estimating the clinical impact, expressed in terms of progression free survival (PFS), associated with the use of the combined strategy (tissue biopsy and liquid biopsy) or the tissue strategy in the EGFR+ mNSCLC population.

METHODS: A pre-existing cost-consequence model was adapted to estimate the annual number of mNSCLC patients with or without the EGFR mutation in order to decide the oncological treatment to be administered in first (1L) or second line (2L). In 1L, against the presence of the EGFR mutation, the administration of a Tyrosine Kinase Inhibitor (TKI), such as osimertinib, gefitinib, erlotinib or afatinib, was considered; in the absence of the EGFR mutation, the administration of standard platinum-based chemotherapy was instead considered. With reference to 2L, in the presence of the EGFR T790M mutation, only osimertinib was considered. In the absence of the EGFR T790M mutation, the administration of the standard platinum-based chemotherapy was also considered. The PFS data associated with each of the drugs considered were extrapolated from the respective clinical studies. Key variables were tested in the sensitivity analysis.

RESULTS: The adoption of the combined strategy (tissue biopsy and liquid biopsy), by virtue of a greater number of patients treated with TKIs, would make it possible to increase the average PFS in the range of 1.1-3,7 months in the $1 \mathrm{~L}$ and by 1.4 months in the $2 \mathrm{~L}$.

CONCLUSION: These results show how the adoption of a correct diagnostic strategy is critical in order to optimize the choice of the therapeutic path in the 1L and $2 \mathrm{~L}$ of mNSCLC. The addition of the liquid biopsy to the classic diagnostic path (tissue biopsy) would in fact allow to obtain an increase in therapeutic efficacy (average PFS).
\end{abstract}

\section{Keywords}

Liquid biopsy; Tissue biopsy; NSCLC; Italian NHS; PFS

\section{INTRODUCTION}

Lung cancer is one of the most frequently diagnosed tumors in Italy ( $11 \%$ of total tumors, excluding skin carcinomas) and, by far, one of the main causes of oncologic death ( $26 \%$ in males and $11 \%$ in females) [1]. The high mortality compared to other tumors is partly due to the delay in the diagnosis. In fact, since the first stages of lung cancer show no symptoms, or show non-specific symptoms, at the time of diagnosis most patients present a locally advanced or metastatic disease [2].

Corresponding author Roberto Ravasio rravasio@aboutpharma.com

Received: 8 November 2019 Accepted: 20 December 2019 Published: 6 February 2020 
Lung cancer is clinically differentiated in two types: Small-Cell Lung Cancer (SCLC, $15 \%$ of cases) and Non-Small Cell Lung Cancer (NSCLC, $85 \%$ of cases) [3].

In recent years, the diagnostic approach to lung cancer has changed radically, requiring in addition to the conventional histopathological diagnosis - the molecular characterization of the tumor (eg. EGFR - Epidermal Growth Factor Receptor, and ALK - Anaplastic Lymphoma Kinase mutations) and some predictors of the response to immunotherapy (eg. PDL1) [4]. Therefore, a histopathological and mutational diagnosis becomes a priority in the correct choice of the most appropriate cancer therapy, especially in the face of a growing availability of target therapies (TT) [5-7]. In the absence of a molecular analysis, the therapeutic choice will in fact be directed towards platinum-based chemotherapy, thus preventing, in the presence of a specific mutation, the benefits deriving from the administration of a TT [5].

A previous analysis [8], which should be referred to for more details, evaluated the economic impact on the Italian National Health Service (NHS) determined by the adoption of different diagnostic strategies that involved the use of tissue biopsy, alone or in combination with the liquid one, to identify the presence of a sensitizing and/or resistance EGFR mutation in the first- and second-line treatment of locally advanced or metastatic non-small cell carcinoma (mNSCLC). The results of the analysis showed that the diagnostic approach characterized by the use of the tissue biopsy alone (tissue strategy) was not cost effective, unlike the one that involved the addition of the liquid biopsy (combined strategy) which, by allowing instead the correct identification of a greater number and type of EGFR mutations, resulted in a lower average cost per correctly identified case.

Based on these previous results - which indirectly also guide the prescription of the best oncological treatment based on the correctly identified mutations - the present analysis was carried out with the aim of estimating the clinical impact, expressed in terms of progression free survival (PFS), associated with the use of the combined strategy (tissue biopsy and liquid biopsy) or the tissue strategy in the EGFR+ mNSCLC population.

\section{METHODS}

\section{Model structure}

A pre-existing Cost-Consequence Model (CCM) was adapted to estimate the annual number of patients with mNSCLC that could be tested for the identification of the EGFR mutation in order to decide the oncological therapy to be administered in first or second line [8]. Specifically, it was verified whether, compared to tissue biopsy alone, the combined strategy could determine - thanks to a greater number of correctly identified EGFR cases, and therefore to a more targeted oncologic therapy - an increase in PFS among mNSCLC patients starting first- or second-line treatment.

Of the original CCM, two diagnostic options were considered, one characterized by the use of tissue biopsy alone (tissue strategy), and the other by the combined use of tissue and liquid biopsy (combined strategy). The tissue strategy (Figure 1), which is mainly the standard of care [8], involves performing only a tissue biopsy for all patients eligible for the diagnostic investigation and for the first-line treatment $(1 \mathrm{~L})$; for the other patients (i.e. poor performance

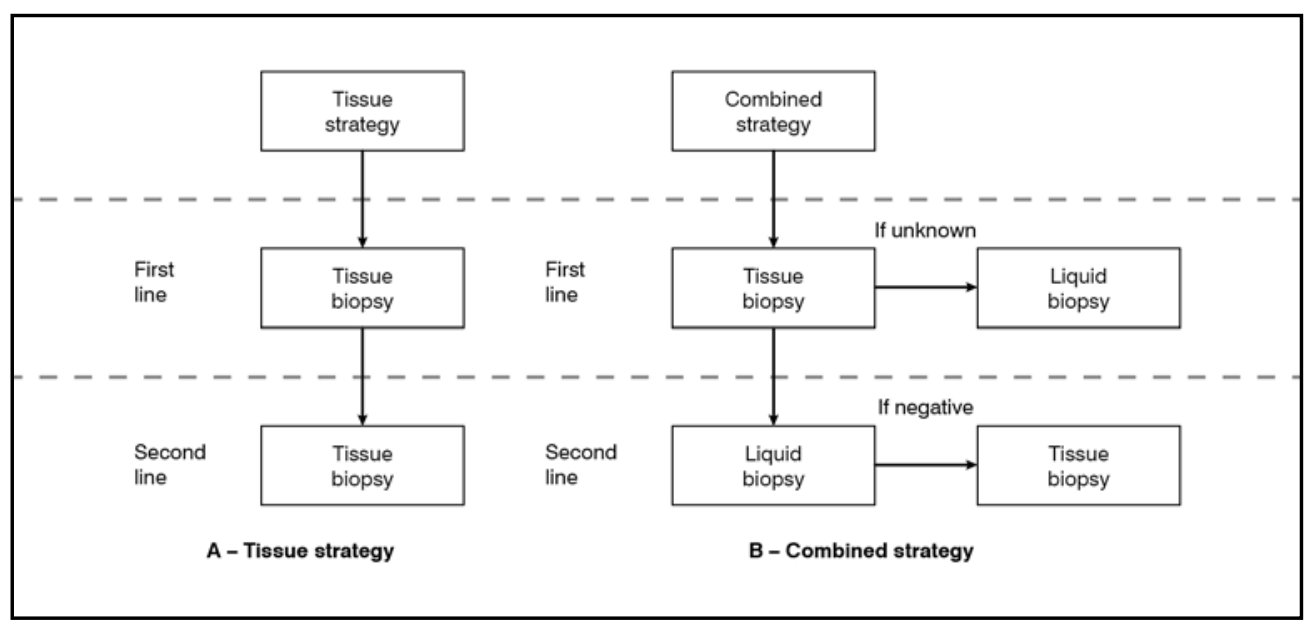

Figure 1. Diagnostic strategies compared in the analysis 


\begin{tabular}{lcc}
\hline \multicolumn{1}{c}{ Parameters } & Population (n) & Source \\
\hline New cases of lung cancer & 41,500 & {$[1]$} \\
Patients with NSCLC (83.0\%) & 34,445 & {$[3]$} \\
Patients with adenocarcinoma, squamous cell carcinoma or large cell carcinoma (67.0\%) & 23,078 & {$[9]$} \\
Patients with locally advanced disease or metastasis at diagnosis (74.1\%) & 17,101 & {$[10]$} \\
First-line treatment & & [3] \\
Patients eligible for the first-line treatment (89.0\%) & 15,220 & Calculated from [12] \\
Patients eligible for the tissue biopsy (85.0\%) & 12,937 & {$[12]$} \\
- determinable outcome after tissue biopsy (70.0\%) & 9,056 & Calculated from [11] \\
- non-determinable outcome after tissue biopsy (30,0\%) & 3,881 & [3] \\
Patients not eligible for tissue biopsy, but eligible for liquid biopsy (15.0\%) & 2,283 & [5] \\
Second-line treatment & 6,840 & Assumption \\
Patients eligible for the second-line treatment (40.0\%) & 5,609 & {$[3]$} \\
Patients eligible for the second tissue biopsy (82.0\%) & 6,840 & {$[13]$} \\
Patients eligible for the liquid biopsy (100.0\%) & & $18.9 \%$ \\
Prevalence of the EGFR mutation & $63.2 \%$ & \\
EGFR+ - first-line treatment & & [3] \\
EGFR+ - second-line treatment
\end{tabular}

Table I. Population included in the CCM

status) - eligible for 1L, but not for tissue biopsy - no further diagnostic procedure is provided for. In case of disease progression (second-line treatment $-2 \mathrm{~L}$ ), the strategy involves performing a second tissue biopsy for all patients eligible for tissue re-biopsy and $2 \mathrm{~L}$. For the other patients eligible for $2 \mathrm{~L}$, but not for tissue re-biopsy, no biopsy is provided. The combined strategy (Figure 1) involves the execution of a tissue biopsy in all patients eligible for the diagnostic investigation and for $1 \mathrm{~L}$; for all cases where it was not possible to determine the outcome after the first tissue biopsy, a liquid biopsy is performed (unlike in the tissue strategy). For the patients eligible for 1L but not for tissue biopsy, no further biopsy procedure is envisaged. Unlike the tissue strategy, in case of disease progression, a liquid biopsy is provided for all patients eligible to receive $2 \mathrm{~L}$. Following a negative outcome of the liquid biopsy in determining the EGFR T790M mutation, a subsequent tissue biopsy is performed.

\section{Population}

The first step of the analysis involved the estimation of the number of patients eligible for the diagnostic strategy (tissue or combined) for the identification of the EGFR mutation (Table I). The estimate was made starting from the number of new cases of lung cancer estimated for 2018 in Italy [1]. From these, the cases of NSCLC [3] were first calculated, and then those with a diagnosis of adenocarcinoma, squamous cell carcinoma or large-cell carcinoma were identified [9]. Only the cases of the subjects with a diagnosis of mNSCLC [10] eligible for $1 \mathrm{~L}$ were then considered [3]. It is estimated that $85 \%$ of these patients are eligible for tissue biopsy [11]. Among patients performing tissue biopsy, the outcome may not be determinable in $30 \%$ of cases [12]. Finally, $40 \%$ of patients with a diagnosis of mNSCLC are eligible to receive $2 \mathrm{~L}$ [3]; of these, $82 \%$ are eligible for tissue re-biopsy [5] and $100 \%$ for liquid biopsy. Based on the literature data, an expected prevalence of the EGFR mutation of $18.9 \%$ and an expected prevalence of the EGFR T790M mutation of $63.2 \%$ were considered for the first-line treatment [3] and for the second-line treatment [13], respectively.

\section{Performance data}

Table II shows the sensitivity and specificity percentages associated with the diagnostic test (cobas ${ }^{\circledR}$ EGFR Mutation Test v2, Roche Molecular Systems, Inc.) performed on tissue samples (tissue biopsy) or plasma (liquid biopsy) for the identification of the EGFR mutation $[14,15]$. The hypothesis un-

\begin{tabular}{lcc}
\hline \multicolumn{1}{c}{ EGFR mutations } & Liquid biopsy & Tissue biopsy \\
\hline First-line treatment $^{1}$ & & \\
Sensitivity (\%) & 95.0 & 98.1 \\
Specificity (\%) & 91.0 & 99.3 \\
Second-line treatment ${ }^{2}$ & & \\
Sensitivity (\%) & 93.0 & 90.0 \\
Specificity (\%) & 92.0 & 98.0 \\
\hline
\end{tabular}

Table II. Sensitivity and specificity associated with the cobas ${ }^{\circledR}$ EGFR Mutation Test $[14,15]$

Exons 19, 21

${ }^{2} \mathrm{~T} 790 \mathrm{M}$ 
derlying the comparison between the two therapeutic strategies is represented by the fact that, by ensuring a greater diagnostic accuracy, the addition of the liquid biopsy makes it possible to identify more frequently the most effective cancer treatment.

\section{Clinical data}

The model, as already mentioned, aimed to estimate the Progression-Free Survival (PFS) of mNSCLC patients on the basis of the diagnostic strategy adopted. Following the AIOM guidelines [16], Table III reports the details of the first- and second-line PFS associated with the cancer therapies currently reimbursed in Italy for the management of mNSCLC patients with or without EGFR mutation. In 1L, against the presence of the EGFR mutation, the administration of a Tyrosine Kinase Inhibitor (TKI), such as osimertinib, gefitinib, erlotinib or afatinib, was considered; in the absence of the EGFR mutation, the administration of standard platinum-based chemotherapy was instead considered. With reference to 2L, in the presence of the EGFR T790M mutation, only osimertinib was considered, in the hypothesis, however, that in the previous first-line treatment only one drug, among gefitinib, erlotinib or afatinib, had been administered. In the absence of the EGFR T790M mutation, the administration of the standard platinum-based chemotherapy was also considered. The PFS data associated with each of the drugs considered were extrapolated from the respective clinical studies [17-27]. In 1L, the PFS with gefitinib, erlotinib or afatinib was calculated as a weighted average based on the patients enrolled in the respective clinical studies [18-26], while for osimertinib the PFS was taken from the FLAURA [17] and AURA3 studies [27], for the first- and second-line treatment, respectively. In comparison with gefitinib, erlotinib and afatinib, the PFS of standard platinum-based chemotherapy in 1L was also calculated as a weighted average (number of patients enrolled) of the respective clinical studies [18-26]. Differently, in the comparison with osimertinib in 1L, not having the FLAURA study [17] estimated a specific data, the PFS associated with the standard platinum-based chemotherapy was calculated as a weighted average (number of patients enrolled) of the PFSs estimated for the chemotherapies considered in the clinical studies conducted with gefitinib, erlotinib and afatinib [18-26]. In 2L, the PFS of osimertinib was compared with that associated with the standard platinum-based chemotherapy, based on the results of the AURA3 study [27].

\section{Model output data}

Based on the number of correctly diagnosed EGFR+ mNSCLC patients, the analysis provided for the two diagnostic strategies, and along a time horizon of one year, the number of patients eligible for treatment (1L and 2L) with TKIs or with platinum-based chemotherapy, and the corresponding difference expressed in terms of average PFS. Specifically, the following comparisons were conducted:

- $1 \mathrm{~L}$

- Osimertinib vs platinum-based chemotherapy

- Gefitinib vs platinum-based chemotherapy

- Erlotinib vs platinum-based chemotherapy

- Afatinib vs platinum-based chemotherapy

\begin{tabular}{|c|c|c|c|}
\hline Indication & Drug & PFS (months) & Source \\
\hline \multicolumn{4}{|c|}{ First-line treatment } \\
\hline \multirow[t]{4}{*}{ EGFR+ } & Osimertinib & 18.9 & FLAURA [17] \\
\hline & Gefitinib & 10.1 & $\begin{array}{l}\text { IPASS [18], First-SIGNAL [19], WJTOG } 3405 \text { [20], } \\
\text { NEJGSG002 [21], LUX-Lung } 7 \text { [22] }\end{array}$ \\
\hline & Erlotinib & 11.3 & OPTIMAL [23], EURTAC [24] \\
\hline & Afatinib & 11.0 & LUX-Lung 3 [25], LUX-Lung 6 [26], LUX-Lung 7 [22] \\
\hline \multirow[t]{4}{*}{ EGFR- } & Chemotherapy vs gefitinib & 6.1 & $\begin{array}{l}\text { IPASS [18], First-SIGNAL [19], WJTOG } 3405 \text { [20], } \\
\text { NEJGSG002 [21], LUX-Lung } 7 \text { [22] }\end{array}$ \\
\hline & Chemotherapy vs erlotinib & 4.9 & OPTIMAL [23], EURTAC [24] \\
\hline & Chemotherapy vs afatinib & 6.2 & LUX-Lung 3 [25], LUX-Lung 6 [26], LUX-Lung 7 [22] \\
\hline & Chemotherapy & 5.9 & Weighted average \\
\hline \multicolumn{4}{|c|}{ Second-line treatment } \\
\hline EGFR + & Osimertinib & 10.1 & AURA3 [27] \\
\hline EGFR- & Chemotherapy & 4.4 & AURA3 [27] \\
\hline
\end{tabular}

Table III. Assumptions for the first- and second-line PFS associated with the treatments considered 
$-2 \mathrm{~L}$

- Osimertinib vs platinum-based chemotherapy

\section{Sensitivity analysis}

The sensitivity analysis was carried out on the parameters most able to affect the results of the base case [28].

With respect to the epidemiological context outlined in the base case, a reduction in the percentage of cases with a non-determinable outcome after the first tissue biopsy was considered, effectively halving - from 30 to $15 \%$ - the probability for patients subsequently subjected to liquid biopsy.

The second parameter, instead, investigated the sensitivity and specificity of the test used to determine the EGFR mutation in the liquid biopsy. In particular, with respect to the base case, the sensitivity and specificity of the test were varied, considering simultaneously (multivariate analysis) the respective lower extremes of the Confidence Interval [15], leaving however unchanged the values of sensitivity and specificity associated with the test for the tissue biopsy.

\section{RESULTS}

With reference to $1 \mathrm{~L}$, the model considered a total of $12,937 \mathrm{mNSCLC}$ patients eligible for the diagnostic investigation (tissue or combined strategy), 2,445 of whom were expected to be EGFR+ (Table IV) [3].

In 2L, eligible mNSCLC patients were instead $6,840,4,325$ of whom were expected to be EGFR T790M+ (Table V) [13]. As an alternative to the tissue strategy, the combined strategy would allow to increase the number of EGFR+ patients correctly diagnosed both in the first $(+679)$ (Table IV) and the second $(+1,054)$ line of treatment (Table V).

The identification of a greater number of EGFR+ patients would lead to an improvement in the clinical benefits expressed in terms of PFS, by virtue of the greater number of patients treated with TKIs. Tables IV and V show, for each of the pharmacological comparisons considered, the average increases in PFS per patient treated, thus favoring the adoption of the combined strategy over the tissue one.

In $1 \mathrm{~L}$ (Table IV), if osimertinib is used, the average increase in PFS would be 3.7 months (from the 14.8 months of the tissue strategy to the 18.5 months of the combined strategy). While considering the administration of the other three TKIs, the average increase in PFS would be in the range of 1.1-1.8 months.

\begin{tabular}{|c|c|c|c|c|c|}
\hline \multirow[t]{3}{*}{ First-line treatment } & \multicolumn{2}{|c|}{ Combined strategy } & \multicolumn{2}{|c|}{ Tissue strategy } & $\underset{\text { (months) }}{\Delta \text { PFS }}$ \\
\hline & \multicolumn{2}{|c|}{12,937} & \multicolumn{2}{|c|}{12,937} & \\
\hline & Patients (n) & PFS (months) & Patients (n) & PFS (months) & \\
\hline \multicolumn{6}{|l|}{ Osimertinib vs chemotherapy } \\
\hline Expected EGFR + & 2,445 & 185 & 2,445 & 14.8 & 3.7 \\
\hline EGFR + correctly identified & 2,376 & 18.9 & 1,679 & 18.9 & \\
\hline EGFR + not correctly identified & 69 & 5.9 & 766 & 5.9 & \\
\hline \multicolumn{6}{|l|}{ Gefitinib vs chemotherapy } \\
\hline Expected EGFR+ & 2,445 & 10.0 & 2,445 & 8.8 & 1.1 \\
\hline EGFR + correctly identified & 2,376 & 10.1 & 1,679 & 10.1 & \\
\hline EGFR + not correctly identified & 69 & 6.1 & 766 & 6.1 & \\
\hline \multicolumn{6}{|l|}{ Erlotinib vs chemotherapy } \\
\hline Expected EGFR + & 2,445 & 11.1 & 2,445 & 9.3 & 1.8 \\
\hline EGFR + correctly identified & 2,376 & 11.3 & 1,679 & 11.3 & \\
\hline EGFR + not correctly identified & 69 & 4.9 & 766 & 4.9 & \\
\hline \multicolumn{6}{|l|}{ Afatinib vs chemotherapy } \\
\hline Expected EGFR+ & 2,445 & 10.9 & 2,445 & 9.5 & 1.4 \\
\hline EGFR + correctly identified & 2,376 & 11.0 & 1,679 & 11.0 & \\
\hline EGFR + not correctly identified & 69 & 6,2 & 766 & 6.2 & \\
\hline
\end{tabular}

Table IV. Results - first-line treatment 


\begin{tabular}{l|cc|cc|c}
\hline \multicolumn{1}{c|}{ Second-line treatment } & \multicolumn{2}{c|}{ Combined strategy } & \multicolumn{2}{c}{ Tissue strategy } & $\begin{array}{c}\Delta \text { PFS } \\
\text { (months) }\end{array}$ \\
\hline Eligible patients $(\mathrm{n})$ & \multicolumn{2}{c}{6,840} & \multicolumn{2}{c}{6,840} \\
\hline \multicolumn{1}{c}{ Osimertinib vs chemotherapy } & Patients (n) & PFS (months) & Patients (n) & PFS (months) & \\
\hline Expected EGFR T790M+ & 4,325 & 10.0 & 4,325 & 8.6 & 1.4 \\
EGFR T790M+ correctly identified & 4,246 & 10.1 & 3,192 & 10.1 & \\
EGFR T790M+ not correctly identified & 79 & 4.4 & 1,133 & 4.4 \\
\hline
\end{tabular}

Table V. Results - second-line treatment

With reference to $2 \mathrm{~L}$ (Table V), the adoption of the combined strategy, once again by virtue of a greater number of patients treated with TKI (osimertinib), would make it possible to increase the average PFS by 1.4 months, from 8.6 months (tissue strategy) to 10.0 months (combined strategy).

\section{Sensitivity analysis}

Table VI reports the results of the sensitivity analysis, expressed in terms of average PFS for each diagnostic strategy adopted. All comparisons confirm the greater clinical effect (increase in average PFS) associated with the combined strategy. Among the two parameters considered, the reduction in the probability that a first-line patient may have a tissue biopsy with a non-determined outcome is the one that mostly affects the results of the comparison between tissue and combined strategy, significantly reducing the difference in the average PFS between the two strategies diagnostic compared to the base case.

\section{DISCUSSION}

The diagnostic/therapeutic approach to NSCLC requires the integration of histopathological diagnosis and molecular characterization of the tumor. In the absence of the molecular analysis, the therapeutic choice will be directed towards platinum-based chemotherapy, precluding the patient, in case of EGFR mutation, from the benefits deriving from the administration (in first and second line) of a targeted therapy (eg. TKIs).

Unlike the tissue biopsy, the liquid biopsy is a low-invasiveness diagnostic method for the evaluation of possible tumor mutations based on the analysis of free circulating DNA (cfDNA) present in the plasma component of the blood [29]. Since blood samples are easily obtained, plasma biopsy is a non-invasive method to supplement traditional biopsy techniques and, in addition, in the advanced therapy lines, it can dynamically evaluate the presence of the most common resistance mechanism to first- and second-generation inhibitors, such as EGFR T790M resistance mutation.

The present analysis evaluated the clinical impact - measured in terms of months free from disease progression - achieved through the adoption of two different diagnostic strate-

\begin{tabular}{|c|c|c|c|}
\hline Parameters & $\begin{array}{c}\text { Combined strategy } \\
\text { (mean PFS) }\end{array}$ & $\begin{array}{c}\text { Tissue strategy } \\
\text { (mean PFS) }\end{array}$ & $\begin{array}{c}\text { Difference } \\
\text { (mean PFS) }\end{array}$ \\
\hline \multicolumn{4}{|c|}{ Cases with a non-determinable outcome after the first tissue biopsy (15\%) } \\
\hline Osimertinib vs chemotherapy (1L) & 18.6 & 16.7 & 1.8 \\
\hline Gefitinib vs chemotherapy (1L) & 10.0 & 9.4 & 0.6 \\
\hline Erlotinib vs chemotherapy (1L) & 11.1 & 10.2 & 0.9 \\
\hline Afatinib vs chemotherapy (1L) & 10.9 & 10.2 & 0.7 \\
\hline Osimertinib vs chemotherapy (2L) & 10.0 & 8.6 & 1.4 \\
\hline \multicolumn{4}{|c|}{ Lower extremes of the $\mathrm{Cl}$ (liquid biopsy sensitivity and specificity) } \\
\hline Osimertinib vs chemotherapy (1L) & 18.4 & 14.8 & 3.6 \\
\hline Gefitinib vs chemotherapy (1L) & 9.9 & 8.8 & 1.1 \\
\hline Erlotinib vs chemotherapy (1L) & 11.1 & 9.3 & 1.8 \\
\hline Afatinib vs chemotherapy (1L) & 10.9 & 9.5 & 1.3 \\
\hline Osimertinib vs chemotherapy (2L) & 9.9 & 8.6 & 1.3 \\
\hline
\end{tabular}

Table VI. Results - sensitivity analysis

$\mathrm{Cl}=$ confidence interval 
gies aimed at determining the EGFR mutation in mNSCLC patients in the first or second line of treatment. The combined approach, which involves the use of the liquid biopsy in addition to the tissue biopsy, allowed - compared to the use of the tissue biopsy alone (tissue strategy) - to identify a greater number of EFGR+ mNSCLC patients. This result, thanks to the possibility of treating a greater number of patients with TKIs, would lead to an increase in the average PFS of EFGR+ mNSCLC patients, depending on the specific TKI administered (1L PFS: 1.1-3.7 months; 2L PFS: 1.4 months).

The results of the present analysis should be read in the light of some observations. The structure of the simulation model adopted here certainly represents a simplification of the current diagnostic-therapeutic pathway for NSCLC, since it is characterized by multiple options and decisions guided by several factors (patient's age, performance status, tolerability, etc.). Despite this, the model has nevertheless considered the treatment options most widely used according to the level of the disease and the treatment lines [16].

The epidemiological analysis was carried out in order to define, starting from a common sample base (number of new cases of lung cancer), the number of patients eligible for the two diagnostic strategies investigated. This process was conducted using data from different bibliographic sources and, in the absence thereof, by making some assumptions. To limit the uncertainty associated with this process, a sensitivity analysis was carried out on the epidemiological variable that could most affect the outcome of the base case: the probability that a patient in $1 \mathrm{~L}$ may have a tissue biopsy with a non-determined outcome. The reduction of this probability from $30 \%$ to $15 \%$, despite not contradicting the results of the base case, would significantly reduce the differential expressed in terms of PFS, to the advantage of the combined strategy.

The sensitivity analysis conducted on the sensitivity and specificity of the test used to identify the EGFR mutation ( $\operatorname{cobas}^{\circledR}$ EGFR Mutation Test v2) largely confirmed the results of the base case.

A further observation also concerns the fact that this model evaluates in particular the hypothesis of a sequential therapeutic strategy with TK inhibitors in relation to the possible appearance of resistance mutation; however, the methodology described can be used in all cases where it is necessary or desirable to perform a biomolecular in-depth analysis in an oncogene-addicted tumor upon the diagnosis and the progression of the disease.

Since they were not present in the literature, it was not possible to compare these results with those of other evaluations, with the sole exception of those of a recent study conducted on the determination of the ALK mutation in NSCLC patients [30]. According to the authors, a greater use of a specific immunohistochemical test (Ventana ${ }^{\circledR}$ ), compared to other tests and FISH, would allow to increase the correct identification of the ALK+ mutation, with a consequent increase in the overall survival from 27.1 months to 32.4 months.

Furthermore, in terms of new diagnostic approaches, liquid biopsy, by allowing the detection of circulating free DNA (cfDNA), paves the way to the use of Next Generation Sequencing (NGS) methodologies, a technique characterized by the possibility of sequencing DNA molecules simultaneously, which allows to identify, in a single analysis: mutations, variations in the number of gene copies, fusions and gene expression. Through the use of NGS panels, a better interpretation of possibly negative investigations for mutations driver of interest is possible. In fact, the identification of at least one gene variant in the liquid biopsy is the proof of the presence of circulating tumor DNA (ctDNA), and allows to report with greater confidence a negative result for the mutation of interest [31].

Finally, it is emphasized that the present analysis just evaluated the clinical consequences deriving from the choice of one of the two different diagnostic strategies, measured in terms of average PFS, net of the economic consequences (treatment cost) which this choice could involve.

\section{CONCLUSIONS}

The possibility to carry out a molecular analysis every time it is necessary, regardless of the patient's performance status, makes it possible to identify the most appropriate cancer therapy, maximizing the effectiveness of the treatment path and minimizing the waste of health resources. The results found here show how the adoption of a correct diagnostic strategy is critical in order to optimize the choice of the therapeutic path in the first- and second-line treatment of mNSCLC. The addition of the liquid biopsy to the classic diagnostic path (tissue biopsy) would in fact allow to obtain an increase in therapeutic efficacy (average PFS). 


\section{Funding}

This research was made possible by an unconditional grant from Roche Diagnostics S.p.A.

\section{Conflict of interest}

GG, LC, and PDP are full-time employees of Roche Diagnostics S.p.A.

RR is a consultant of Health Publishing \& Services, which has received an unconditional

grant by Roche Diagnostics S.p.A. for the conduct of the research.

DC declares that he has no conflicts of interest in this research.

\section{REFERENCES}

1. AIOM, AIRTUM e Fondazione AIOM. I numeri del cancro in Italia 2018. Available at: https://www.aiom.it/wp-content/uploads/2018/10/2018_NumeriCancro-operatori.pdf(last accessed September 2019)

2. Lim C, Sung M, Shepherd FA, et al. Patients with Advanced Non-Small Cell Lung Cancer: Are Research Biopsies a Barrier to Participation in Clinical Trials?. J Thorac Oncol 2016; 11: 79-84; https://doi.org/10.1016/j.jtho.2015.09.006

3. Gobbini E, Galetta D, Tiseo M, et al. Molecular profiling in Italian patients with advanced non-small-cell lung cancer: An observational prospective study. Lung Cancer 2017; 111: 30-7; https://doi.org/10.1016/j.lungcan.2017.06.009

4. Chan BA, Hughes BG. Targeted therapy for non-small cell lung cancer: current standards and the promise of the future. Transl Lung Cancer Res 2015; 4: 36-54; https://doi.org/10.3978/j. issn.2218-6751.2014.05.01

5. Chouaid C, Dujon C, Do P, et al. Feasibility and clinical impact of re-biopsy in advanced non small-cell lung cancer: a prospective multicenter study in a real-world setting (GFPC study 12-01). Lung Cancer 2014; 86: 170-3; https://doi.org/10.1016/j.lungcan.2014.08.016

6. Ellis PM, Blais N, Soulieres D, et al. A systematic review and Canadian consensus recommendations on the use of biomarkers in the treatment of non-small cell lung cancer. J Thorac Oncol 2011; 6: 1379-91; https://doi.org/10.1097/JTO.0b013e318220cb8e

7. Peters S, Adjei AA, Gridelli C, et al. Metastatic non-small-cell lung cancer (NSCLC): ESMO Clinical Practice Guidelines for diagnosis, treatment and follow-up. Ann Oncol 2012; 23 Suppl 7: vii56-64; https://doi.org/10.1093/annonc/mds226

8. Gancitano G, Ravasio R, Dionisi M, et al. Cost-Consequence Analysis of Three Different Diagnostic Strategies in the First- and Second-Line Treatment of Locally Advanced or Metastatic Non-Small-Cell Lung Cancer. Farmeconomia. Health economics and therapeutic pathways 2018; 19: 27-35; https://doi.org/10.7175/fe.v19i1.1354

9. AIOM, AIRTUM e Fondazione AIOM. I numeri del cancro in Italia 2016. Available at: http://www.registri-tumori.it/PDF/AIOM2016/I_numeri_del_cancro_2016.pdf(last accessed January 2018)

10. Draheim S, Eisinger B, Förster S, et al. Krebsinzidenz und Krebsmortalität 2009-2012. Available at: https://www.berlin.de/gkr/_assets/jahresbericht-2009-2012.pdf(last accessed January 2018)

11. Lim C, Sung M, Shepherd FA, et al. Patients with Advanced Non-Small Cell Lung Cancer: Are Research Biopsies a Barrier to Participation in Clinical Trials?. J Thorac Oncol 2016; 11: 79-84; https://doi.org/10.1016/j.jtho.2015.09.006

12. Malapelle U, Sirera R, Jantus-Lewintre E, et al. Profile of the Roche cobas ${ }^{\circledR}$ EGFR mutation test v2 for non-small cell lung cancer. Expert Rev Mol Diagn 2017; 17: 209-15; https://doi. org/10.1080/14737159.2017.1288568

13. Yoon HJ, Lee HY, Lee KS, et al. Repeat biopsy for mutational analysis of non-small cell lung cancers resistant to previous chemotherapy: adequacy and complications. Radiology 2012; 265: 939-48; https://doi.org/10.1148/radiol.12112613

14. Jenkins S, Yang JC, Ramalingam SS, et al. Plasma ctDNA Analysis for Detection of the EGFR T790M Mutation in Patients with Advanced Non-Small Cell Lung Cancer. J Thorac Oncol 2017; 12: 1061-70; https://doi.org/10.1016/j.jtho.2017.04.003

15. Cheng MM, Palma JF, Scudder S, Poulios N, Liesenfeld O. The Clinical and Economic Impact of Inaccurate EGFR Mutation Tests in the Treatment of Metastatic Non-Small Cell 
Lung Cancer. J Pers Med 2017; 7: 5; https://doi.org/10.3390/jpm7030005

16. Associazione Italiana di Oncologia Medica - AIOM. Linee guida neoplasie del polmone. Edizione 2019.Available at: https://www.aiom.it/wp-content/uploads/2019/10/2019_LG_ AIOM_Polmone-1.pdf (last accessed December 2019)

17. Soria JC, Ohe Y, Vansteenkiste J, et al. Osimertinib in Untreated EGFR-Mutated Advanced Non-Small-Cell Lung Cancer. N Engl J Med 2018; 378: 113-25; https://doi.org/10.1056/ NEJMoa1713137

18. Mok TS, Wu YL, Thongprasert S, et al. Gefitinib or carboplatin-paclitaxel in pulmonary adenocarcinoma. $N$ Engl J Med 2009; 361: 947-57; https://doi.org/10.1056/NEJMoa0810699

19. Han JY, Park K, Kim SW, et al. First-SIGNAL: first-line single-agent iressa versus gemcitabine and cisplatin trial in never-smokers with adenocarcinoma of the lung. $J$ Clin Oncol 2012; 30: 1122-8; https://doi.org/10.1200/JCO.2011.36.8456

20. Mitsudomi T, Morita S, Yatabe Y, et al. Gefitinib versus cisplatin plus docetaxel in patients with non-small-cell lung cancer harbouring mutations of the epidermal growth factor receptor (WJTOG3405): an open label, randomised phase 3 trial. Lancet Oncol 2010; 11: 121-8; https://doi.org/10.1016/S1470-2045(09)70364-X

21. Maemondo M, Inoue A, Kobayashi K, et al. Gefitinib or chemotherapy for non-small-cell lung cancer with mutated EGFR. $N$ Engl J Med 2010; 362: 2380-8; https://doi.org/10.1056/ NEJMoa0909530

22. Park K, Tan EH, O'Byrne K, et al. Afatinib versus gefitinib as first-line treatment of patients with EGFR mutation-positive non-small-cell lung cancer (LUX-Lung 7): a phase 2B, open-label, randomised controlled trial. Lancet Oncol 2016; 17: 577-89; https://doi. org/10.1016/S1470-2045(16)30033-X

23. Zhou C, Wu YL, Chen G, et al. Erlotinib versus chemotherapy as first-line treatment for patients with advanced EGFR mutation-positive non-small-cell lung cancer (OPTIMAL, CTONG-0802): a multicentre, open-label, randomised, phase 3 study. Lancet Oncol 2011; 12: 735-42; https://doi.org/10.1016/S1470-2045(11)70184-X

24. Rosell R, Carcereny E, Gervais R, et al. Erlotinib versus standard chemotherapy as firstline treatment for European patients with advanced EGFR mutation-positive non-small-cell lung cancer (EURTAC): a multicentre, open-label, randomised phase 3 trial. Lancet Oncol 2012; 13: 239-46; https://doi.org/10.1016/S1470-2045(11)70393-X

25. Sequist LV, Yang JC, Yamamoto N, et al. Phase III study of afatinib or cisplatin plus pemetrexed in patients with metastatic lung adenocarcinoma with EGFR mutations. $J$ Clin Oncol 2013; 31: 3327-34; https://doi.org/10.1200/JCO.2012.44.2806

26. Wu YL, Zhou C, Hu CP, et al. Afatinib versus cisplatin plus gemcitabine for first-line treatment of Asian patients with advanced non-small-cell lung cancer harbouring EGFR mutations (LUX-Lung 6): an open-label, randomised phase 3 trial. Lancet Oncol 2014; 15: 213-22; https://doi.org/10.1016/S1470-2045(13)70604-1

27. Mok TS, Wu Y-L, Ahn M-J, et al. Osimertinib or Platinum-Pemetrexed in EGFR T790M-Positive Lung Cancer. $N$ Engl J Med 2017; 376: 629-40; https://doi.org/10.1056/ NEJMoa1612674

28. AIES - Associazione Italiana di Economia Sanitaria. Proposte di linee guida per la valutazione economica degli interventi sanitari. Pharmacoeconomics Italian Research Articles 2009; 11: 89-93

29. Diaz LA Jr, Bardelli A. Liquid biopsies: genotyping circulating tumor DNA. J Clin Oncol 2014; 32: 579-86; https://doi.org/10.1200/JCO.2012.45.2011

30. Paolini D, Tiseo M, Demma F, et al. Ventana ALK (D5F3) in the Detection of Patients Affected by Anaplastic Lymphoma Kinase-positive Non-Small-cell Lung Cancer: Clinical and Budget Effect. Clin Lung Cancer 2018; 19: e735-43; .https://doi.org/10.1016/j. cllc.2018.05.012

31. Gruppo di Lavoro AIOM - SIAPEC-IAP - SIF - SIBioC. Raccomandazioni per l'esecuzione di test molecolari su biopsia liquida in oncologia 2018. Available at https://www.aiom. it/wp-content/uploads/2018/09/2018_biopsialiquida.pdf (last accessed September 2019) 